\title{
Involvement of the rat anterior cingulate cortex in control of instrumental responses guided by reward expectancy
}

\author{
Judith Schweimer and Wolfgang Hauber ${ }^{1}$ \\ Department of Animal Physiology, Institute of Biology, University of Stuttgart, Stuttgart D-70550, Germany
}

\begin{abstract}
The anterior cingulate cortex (ACC) plays a critical role in stimulus-reinforcement learning and reward-guided selection of actions. Here we conducted a series of experiments to further elucidate the role of the ACC in instrumental behavior involving effort-based decision-making and instrumental learning guided by reward-predictive stimuli. In Experiment 1, rats were trained on a cost-benefit T-maze task in which they could either choose to climb a barrier to obtain a high reward (four pellets) in one arm or a low reward (two pellets) in the other with no barrier present. In line with previous studies, our data reveal that rats with quinolinic acid lesions of the ACC selected the response involving less work and smaller reward. Experiment 2 demonstrates that breaking points of instrumental performance under a progressive ratio schedule were similar in sham-lesioned and ACC-lesioned rats. Thus, lesions of the ACC did not interfere with the effort a rat is willing to expend to obtain a specific reward in this test. In a subsequent task, we examined effort-based decision-making in a lever-press task where rats had the choice between pressing a lever to receive preferred food pellets under a progressive ratio schedule, or free feeding on a less preferred food, i.e. lab chow. Results show that sham- and ACC-lesioned animals had similar breaking points and ingested comparable amounts of less-preferred food. Together, the results of Experiment 1 and 2 suggest that the ACC plays a role in evaluating how much effort to expend for reward; however, the ACC is not necessary in all situations requiring an assessment of costs and benefits. In Experiment 3 we investigated learning and reversal learning of instrumental responses guided by reward predictive stimuli. A reaction time (RT) task demanding conditioned lever release was used in which the upcoming reward magnitude (five vs. one food pellet) was signalled in advance by discriminative visual stimuli. Results revealed that rats with ACC lesions were able to discriminate reward magnitude-predictive stimuli and to adapt instrumental behavior to reversed stimulus-reward magnitude contingencies. Thus, in a simple discrimination task as used here, the ACC appears not to be required to discriminate reward magnitude-predictive stimuli and to use the learned significance of the stimuli to guide instrumental behavior.
\end{abstract}

\begin{abstract}
The rat anterior cingulate cortex (ACC) is an integral part of the prefrontal cortex (Paxinos and Watson 1997) lying on its medial surface caudal to the genu of the corpus callosum with interconnections to numerous regions including the caudate nucleus (McGeorge and Faull 1989), nucleus accumbens (ACB) (Berendse et al. 1992; Brog et al. 1993), ventral tegmental area (VTA), mediodorsal nucleus of the thalamus (Uylings and van Eden 1990), and amygdala (Divac and Diemer 1980).

The ACC has been implicated in learning to discriminate multiple visual stimuli on the basis of the association of these stimuli with reward. Accordingly, lesions of the ACC impaired performance of rats preferentially in complex tasks including eight-pair concurrent visual discrimination (Bussey et al. 1997b), a two-stimulus temporally discriminated approach task (Cardinal et al. 2003), and autoshaping (Bussey et al. 1997a; Parkinson et al. 2000; Cardinal et al. 2002, 2003). In contrast, lesions of the ACC did not interfere with performance in simpler tasks such as onepair concurrent visual discrimination (Bussey et al. 1997b), and even improved performance in some tasks (Bussey et al. 1996). From these and other studies it has been concluded that the ACC plays an important role in mediating instrumental behaviors that depend on stimulus-reinforcer contingencies and require discrimination of relatively similar stimuli. In particular, the ACC
\end{abstract}

\section{'Corresponding author.}

E-mail hauber@bio.uni-stuttgart.de; fax 49 711-685-5090.

Article and publication are at http://www.learnmem.org/cgi/doi/10.1101/ Im.90605. could represent a 'disambiguating' structure enhancing stimulus discrimination, if stimuli share common elements, i.e., are in the same sensory modality and are similar (Cardinal et al. 2003).

In addition, the ACC has been implicated in effort-based decision-making. Rats with ACC lesions tested in a cost-benefit T-maze task no longer preferred the effortful high-reward action; rather they preferred the less effortful low-reward action (Walton et al. 2003). Thus, ACC functions might be essential for decisions regarding whether it is worth making effortful actions given the value of the expected outcome (Rushworth et al. 2004). On the other hand, lesions of the ACC did not impair the high preference of rats for large, delayed rewards over immediate, low rewards in a delayed reinforcement choice task (Cardinal et al. 2001). These findings raise the possibility that the ACC is not necessary in all decisions involving cost-benefit analysis. Major variables influencing effort-based decisions are only partially known, and their impact is not fully understood. For instance, it is likely that response costs may include not only force requirements or caloric expenditure, but probably also skill or temporal requirements (Salamone et al. 2003). Thus, it remains to be further specified which kind of effort-based decisions depend on the ACC.

The aim of the present study was to characterize the role of the ACC (including the Cg1 and Cg2 fields of the ACC) in stimulus-reward learning and effort-based decision-making in more detail. In Experiment 1, rats were trained in a T-maze cost-benefit task (Walton et al. 2003) in which they could either choose to climb a barrier $(25 \mathrm{~cm})$ to obtain a high reward (four pellets) in 
one arm of the maze or a low reward (two pellets) in the other arm with no barrier present. In Experiment 2, the same rats were tested in a modified lever-press task (Salamone et al. 1991) in which they had the choice between pressing a lever to receive preferred food pellets on a progressive ratio (PR) schedule, or free feeding on a less preferred food, i.e., lab chow. If the ACC plays a general role in effort-based decisions, rats with ACC lesions should be impaired in both kinds of cost-benefit tasks. In Experiment 3, another group of rats was examined in a reaction time (RT) task demanding conditioned lever release in which the upcoming reward magnitude (five vs. one food pellet) was signalled in advance by discriminative stimuli. Usually, intact rats rapidly discriminate reward magnitude-predictive stimuli and respond faster for expected high reward within a few days (e.g., Bohn et al. 2003a; Giertler et al. 2003). In this task, visual stimuli which predict reward magnitudes differ only in their brightness, i.e., they are in the same modality and not easy to discriminate. If the ACC is involved to facilitate discrimination of similar conditioned stimuli, rats with ACC lesions should be impaired in this discrimination task.

\section{Experiment 1: The role of the ACC in effort-based decision making in a T-maze task}

\section{Results}

\section{Histology}

In sham-lesioned animals $(n=9)$ we observed minimal damage caused by the injection cannulae during vehicle microinfusion. In animals with quinolinic acid (QA) lesions of the ACC $(n=8)$, neuronal loss and gliosis was prominent in fields Cg1 and 2 with a maximum extension from $\sim 2.7 \mathrm{~mm}$ anterior to bregma to 1.3 $\mathrm{mm}$ posterior to bregma (Fig. 1). Lesions included predominantly pregenual and perigenual parts of the ACC; minor damage of the prelimbic cortex was detected occasionally, and the corpus callosum was generally spared. One animal was excluded from analysis as the lesion was too small.

\section{Behavior}

In the pre-lesion testing block, all animals exhibited a strong preference to surmount the barrier to obtain the high reward (HR) with an average of $\sim 90 \%$ of the choices for the HR (Fig. 2). This preference was reduced postoperatively in QA-lesioned rats, when tested in the one-barrier situation, but not when tested in the two-barrier situation. Repeated measures three-way ANOVA revealed significant effects of treatment $\left[F_{(1,15)}=7.35, P<0.05\right]$ (between-subjects factor) as well as testing blocks $\left[F_{(3,45)}=9.96\right.$, $P<0.001]$ and days $\left[F_{(2,30)}=5.00, P<0.05\right]$ (within-subjects factors). Furthermore, there was a significant treatment $\times$ block interaction $\left[F_{(3,45)}=5.26, P<0.01\right]$. In the pre-lesion testing block, no performance differences in animals to be sham- or QAtreated were determined by means of post hoc analysis $\left[F_{(1,15)}=0.024, P=0.87\right]$. In post-lesion testing blocks, ACC- as well as sham-lesioned animals were able to climb the barrier on forced trials, indicating the absence of sensorimotor impairments. Most importantly, ACC-lesioned animals exhibited a reduced preference for the HR with barrier $\left[F_{(1,15)}=5.96, P<0.05\right]$ in the one-barrier situation. However, when the second barrier was introduced into the low-reward condition (LR), all rats chose the $\operatorname{HR}\left[F_{(1,15)}=0.55, P=0.47\right]$. After the barrier in the LR was removed in the next testing block, the ACC-lesioned animals no longer preferred the $\operatorname{HR}\left[F_{(1,15)}=7.96, P<0.05\right]$.

\section{Discussion}

The histological and behavioral results of Experiment 1 confirm earlier findings (Walton et al. 2003) and demonstrate that rats

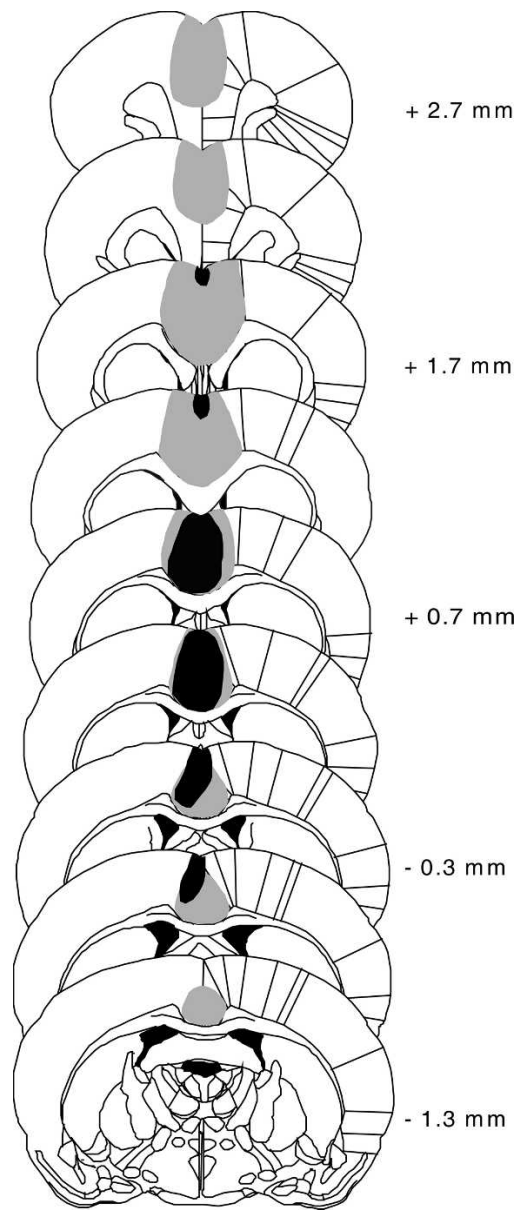

Figure 1. Schematic representation of the minimum (black shading) and maximum (gray shading) extension of ACC lesion in rats used in Experiments 1 and $2(n=8)$. Adapted with permission from Elsevier (C) 1997, Paxinos and Watson (1997). Scales are relative to bregma.

with ACC lesions were impaired in effort-based decision-making in a T-maze cost-benefit task. ACC-lesioned animals had a reduced preference for the HR option and preferentially selected the LR without a barrier. However, if ACC-lesioned rats had to surmount a barrier for either reward, they preferred the HR arm.

The placement and size of ACC lesions in the present experiment is in keeping with a study of Walton et al. (2003), who used the same dosing regimen of QA. Likewise, we observed that ACC-lesioned rats no longer preferred climbing the barrier for a higher reward in the one-barrier condition, but were still capable of climbing a barrier and remembering the HR location in the two-barrier condition. The reduced preference of the HR in the one-barrier condition was a reproducible effect, as removal of the barrier in the LR reinstated the LR preference in ACC-lesioned animals. Minor differences between both studies are also discernible. ACC lesions in our rats extended to more posterior parts of the ACC. In addition, in our experiment sham-lesioned rats still displayed a preference for the HR in the one-barrier condition, whereas those in the study of Walton et al. (2003) performed at chance level. Moreover, in our experiment ACC-lesioned animals had a higher preference for the HR arm in the two-barrier condition compared to those in the Walton et al. (2003) study. It is likely that the lower barrier $(25 \mathrm{~cm}$ instead of $30 \mathrm{~cm})$ used in our protocol accounts for these differences.

The impairment in ACC-lesioned animals could reflect an impaired ability to integrate expected costs and benefits of an 


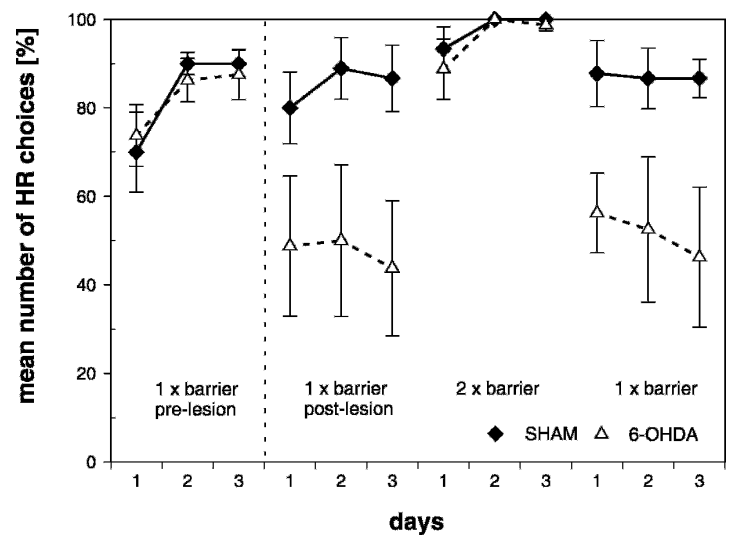

Figure 2. Effects of ACC lesions in a T-maze cost-benefit task. Mean ( \pm SEM) percentage of HR choices per day in sham-lesioned $(n=9$, filled rectangles) and ACC-lesioned ( $n=8$, open triangles) rats are given. Testing block 1 was pre-lesion, blocks 2-4 post-lesion. Each testing block consisted of three consecutive test days. On blocks 1,2 , and 4 , a $25-\mathrm{cm}$ barrier was placed in the HR; on block 3 identical $25-\mathrm{cm}$ barriers were placed in the $H R$ and $L R$, respectively.

action rather than an insensitivity to different reward quantities, failure to remember the size or position of a reward, or a motor impairment to climb the barrier (Walton et al. 2002, 2003). Our data confirm this view, as animals with ACC lesions were not insensitive to costs and benefits and modified their choice behavior in the two-barrier condition. It appears that a hypothetical decision criterion or value function underlying the costbenefit analysis may have been shifted in ACC-lesioned animals. However, the variables influencing effort-based decisions as tested here are not well understood. For instance, it is likely that response costs may include not only force requirements or caloric expenditure, but probably also skill and temporal requirements or cognitive aspects influencing perceived task difficulty (Salamone et al. 2003). Despite these limitations, the results of Experiment 1 correspond with the view that the ACC is critically involved in evaluating how much effort to expend for a specific reward. This notion is supported by primate electrophysiological studies demonstrating that neurons in the ACC play a role in processing reward information for motor selection (Shima and Tanji 1998). In addition, ACC lesions, while not interfering with visual discrimination learning or performance, impaired selection of responses associated with different rewards (Hadland et al. 2003).

\section{Experiment 2: The role of the ACC in effort-based decision-making in a lever-press task}

\section{Results}

\section{Behavior}

Rats used in Experiment 1 were subsequently tested in lever-press tasks in operant boxes involving a progressive ratio (PR) schedule. The highest ratio of responding (lever presses per reward) achieved in a PR schedule is termed the "breaking point." As shown in Figure 3, rats with ACC lesions reached a breaking point similar to that of the sham-lesioned animals, i.e., about 60 lever presses per pellet. ANOVA showed a significant main effect of days $\left[F_{(9,135)}=7.78, P<0.001\right]$, but not of treatment $\left[F_{(1,15)}=0.29, P=0.60\right]$ and no treatment $\times$ testing day interaction $\left[F_{(9,135)}=0.43, P=0.91\right]$. Likewise, the latency to achieve the breaking point and the number of perseverative lever presses, i.e., extra lever presses after reward was signalled, was not altered in
ACC-lesioned rats. An ANOVA for either condition revealed no significant effects (data not shown). Thereafter, animals were examined in a cost-benefit test in which they had the choice to work under a PR schedule for preferred food or obtain freely available lab chow. A free choice test revealed that sham- and ACC-lesioned animals had a preference for food pellets over lab chow (data not shown). In sham- and ACC-lesioned animals, breakpoints were decreased to about 10 lever presses per pellet in the choice test relative to the preceding test with a PR schedule without access to lab chow (Fig. 4). ANOVA revealed no effect of treatment $\left[F_{(1,30)}=0.21, P=0.65\right]$, but a significant effect of test condition (lab chow available vs. not available) $\left[F_{(1,30)}=41.74\right.$, $P<0.001]$. In addition, sham- and ACC-lesioned animals ingested a similar amount of lab chow $\left[t_{(15)}=-1.25, P=0.23\right]$ in the choice test.

\section{Discussion}

Experiment 2 reveals that lesions of the ACC did not interfere with the animals' motivation to work for food as indicated by their intact performance on a PR schedule. In addition, effortbased decision-making was not impaired in a cost-benefit task with the options to get preferred food pellets under a PR schedule or freely available but less preferred lab chow.

In the instrumental task involving a PR schedule without access to lab chow, sham- and ACC-lesioned animals had similar breaking points. The breaking point is generally thought to represent a measure of how much effort an animal is willing to expend for a certain reward (e.g., Hodos 1961; Bowman and Brown 1998; Baunez et al. 2002). Therefore, ACC-lesioned animals were not insensitive to evaluating increasing costs relative to a constant benefit in general, as already noted in the twobarrier condition in Experiment 1. However, these tasks are difficult to compare, as instrumental performance on a PR schedule does not include a choice between two response options as in the T-maze task.

In a subsequent test using a cost-benefit choice test with lab chow freely available, breaking points in sham- and ACClesioned animals were reduced to about the same extent, and they ingested similar amounts of lab chow. This result was unexpected, as it indicates that ACC functions are not essential for intact cost-benefit choice behavior in this task. Together, the results of Experiment 1 and 2 suggest that the ACC is apparently not necessary for all decisions involving cost-benefit analysis. This view is also supported by findings that lesions of the ACC did not impair the high preference for large delayed rewards over

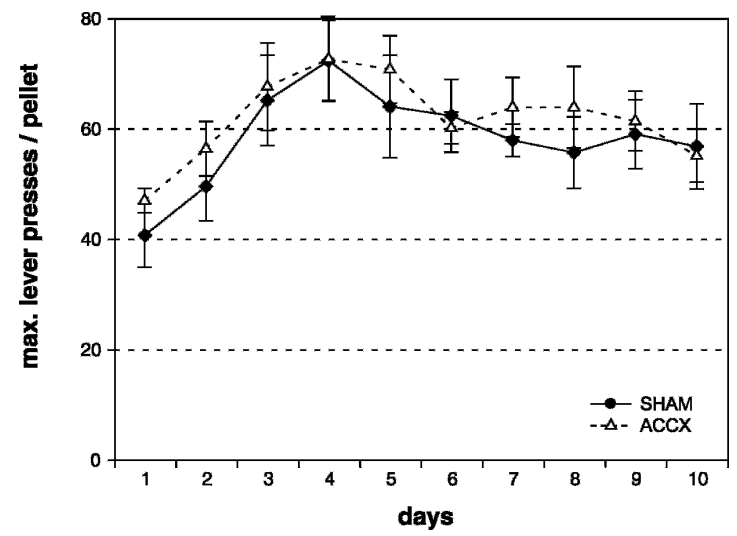

Figure 3. Effects of $\mathrm{ACC}$ lesions on instrumental responding under a progressive ratio schedule. Breaking points, i.e., mean lever presses per pellets ( \pm SEM) of highest ratio during daily sessions from sham- $(n=9$, filled rectangles) and ACC-lesioned ( $n=8$, open triangles) rats are given. 


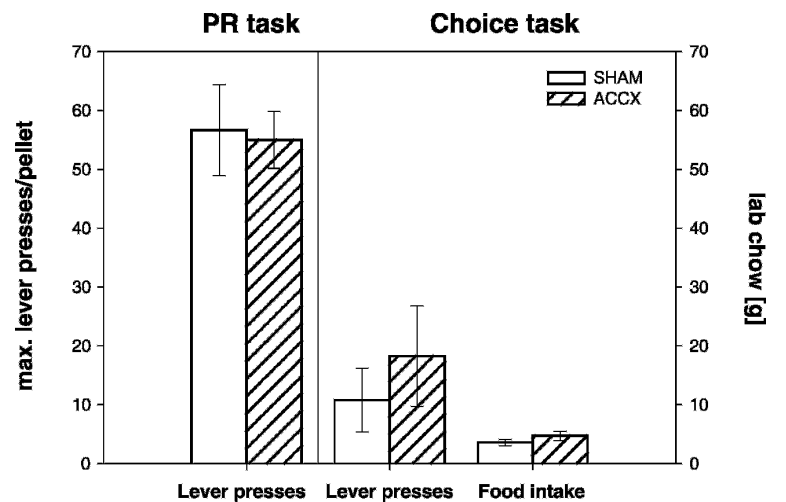

Figure 4. Effects of $A C C$ lesions on instrumental responding for food pellets under a progressive ratio (PR) schedule with or without concurrent access to less-preferred lab chow. Breaking points, i.e., mean lever presses per pellets $( \pm$ SEM) of highest ratio during daily sessions as well as the amount of ingested lab chow are depicted. The left panel shows breaking points on Day 10, when rats were tested under a PR schedule without access to lab chow. The right panel shows breaking points when rats were tested under a PR schedule with access to lab chow and the amount of lab chow ingested. Open bars, sham-lesioned animals $(n=9)$; hatched bars, ACC-lesioned rats $(n=8)$.

immediate low rewards in a delayed reinforcement choice test (Cardinal et al. 2001). From our experiments it is difficult to identify attributes of those effort-based decisions that depend on the ACC, as the tasks in Experiments 1 and 2 differ in various aspects. For instance, the T-maze test requires a decision between two instrumental actions, i.e., crossing a barrier or running an alley, whereas the operant-box task requires a decision between an instrumental behavior, i.e., lever pressing, and chow consumption, which might represent consummatory rather than instrumental behavior (Salamone et al. 1997). In addition, other variables covary such as the type and attractiveness of food reward. Moreover, the choice behavior in Experiment 1 was tested in repeated sessions over subsequent days, whereas in Experiment 2 only a single test session was used.

\section{Experiment 3: ACC and discrimination of reward-predictive stimuli in a reaction time task}

\section{Results}

\section{Histology}

As in Experiment 1, sham-lesioned animals $(n=14)$ used in this experiment exhibited little damage caused by the injection cannulae. In contrast, neuronal loss and gliosis in ACC-lesioned animals $(n=14)$ was prominent, with a maximum extent of $\sim 2.7$ $\mathrm{mm}$ anterior to bregma to $1.3 \mathrm{~mm}$ posterior to bregma (Fig. 5). Lesions included pregenual and perigenual parts of the ACC, and occasionally there was small damage of the prelimbic cortex; the corpus callosum was generally spared.

\section{Behavior: Acquisition}

On completion of postoperative acquisition, RTs were significantly shorter for expected high compared to low reward. An ANOVA revealed significant main effects of reward magnitude $\left[F_{(1,26)}=47.28, P<0.001\right]$ and days $\left[F_{(13,338)}=3.44, P<0.001\right]$, but not of treatment $\left[F_{(1,26)}=0.06, P=0.81\right]$. Furthermore, there was a significant days $\times$ reward magnitude interaction $\left[F_{(13,338)}=2.78, P<0.001\right]$. Sham-lesioned animals exhibited significant shorter RTs from Day 2 on, ACC-lesioned animals on Day 2 and on Day 5 and beyond (Fig. 6). Accuracy of perfor- mance increased during the course of acquisition, in particular if high reward was expected (data not shown). The ANOVA on accuracy of performance showed significant main effects of reward magnitude $\left[F_{(1,26)}=24.79, P<0.001\right]$ and days $\left[F_{(13,338)}=4.98, P<0.001\right]$, but not of treatment.

\section{Behavior: Reversal}

After a shift of the original stimulus-reward magnitude contingencies, the RTs of sham-lesioned and ACC-lesioned animals did not differ significantly (Fig. 7). Guidance of RTs by expected reward changed over a period of 9-10 d, and thereafter shamlesioned animals and ACC-lesioned animals significantly discriminated the novel stimulus-reward magnitude contingencies. An ANOVA revealed no significant main effect of treatment $\left[F_{(1,26)}=0.02, P=0.90\right]$ and reward magnitude $\left[F_{(1,26)}=0.09\right.$, $P=0.77]$, but a significant main effect of days $\left[F_{(9,234)}=4.94\right.$, $P<0.001]$. Furthermore, a significant day $\times$ reward magnitude interaction was detected $\left[F_{(9,234)}=20.21, P<0.001\right]$.

\section{Discussion}

The result of Experiment 3 demonstrate that lesions of the ACC did not impair discriminative guidance of instrumental responses by reward magnitude-predictive stimuli. During initial learning and during learning a reversal of original stimulusreward magnitude contingencies, the RTs of ACC- and shamlesioned rats were eventually guided by stimulus-reward magni-

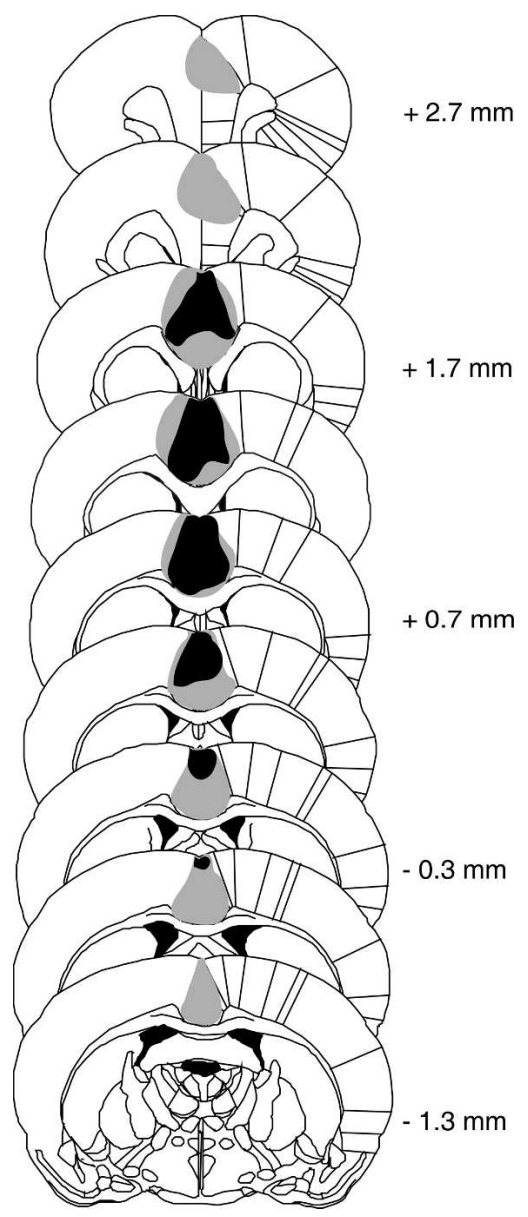

Figure 5. Schematic representation of the minimum (black shading) and maximum (gray shading) extension of ACC lesion of rats $(n=14)$ used in Experiment 3. Adapted with permission from Elsevier (C) 1997, Paxinos and Watson (1997). Scales are relative to bregma. 

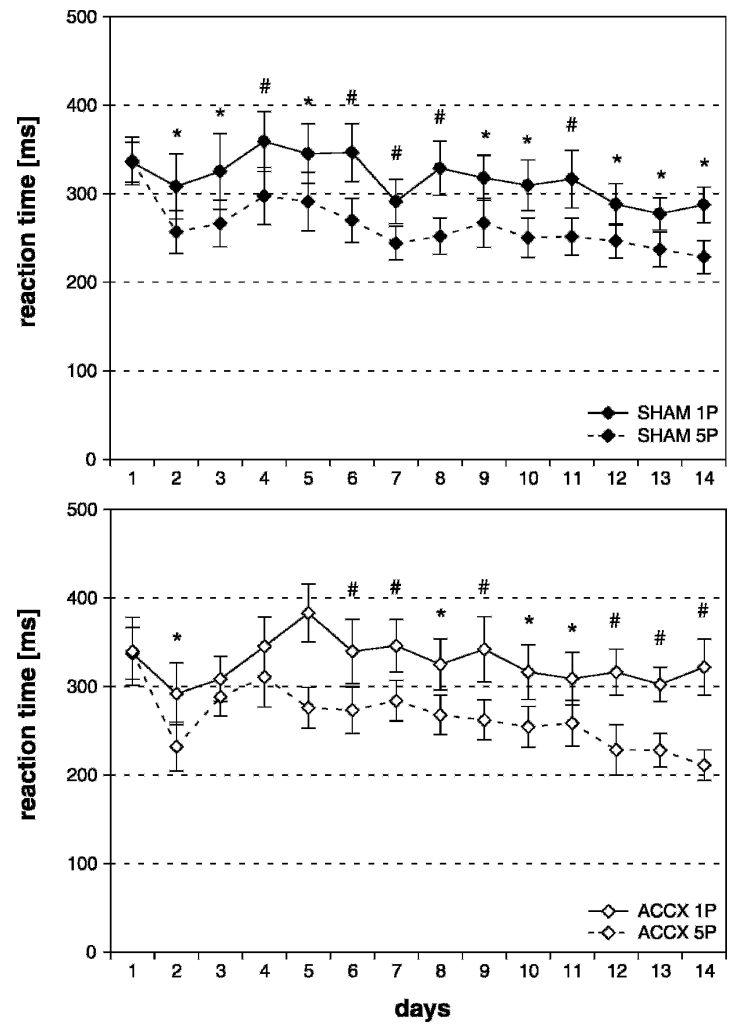

Figure 6. Effects of ACC lesions on discrimination of reward magnitude-predictive stimuli during acquisition of a reaction time (RT) task. Mean ( \pm SEM) of RTs from correct trials for expected high (five pellets) and low reward (one pellet) in sham-lesioned animals $(n=14)(A)$ and ACC-lesioned animals $(n=14)(B)$ are given. Solid line, RTs for five pellets; dotted line, RTs for one pellet. ${ }^{*} P<0.05, \# P<0.01$ : significant differences in RTs for expected high vs. low reward on individual days.

tude contingencies. Therefore, the ACC may not be necessary in simple discrimination problems involving only two reward predictive stimuli as used here. Other prefrontal subregions such as the orbitofrontal cortex appear to contribute to reversal learning in this task (Bohn et al. 2003b). Accordingly, Bussey et al. (1997b) found that rats with ACC lesions were not impaired in a one-pair concurrent discrimination task, but exhibited deficits in performing an eight-pair concurrent discrimination task.

As ACC lesions impaired performance particularly in tasks with multiple conditioned stimuli, it has been suggested that the ACC 'disambiguates' conditioned stimuli and prevents stimulus generalization (Cardinal et al. 2002). However, ACC-lesioned animals were able to discriminate stimuli of different modalities and visual stimuli differing in a primary submodality such as color (Bussey et al. 1997b; Cardinal et al. 2002). Therefore, 'disambiguation' by the ACC might be particularly relevant if two conditioned stimuli to be discriminated share common elements (Cardinal et al. 2003). Our data are at variance with this assumption, because ACC-lesioned animals discriminated rewardpredictive stimuli of the same modality that differ in brightness only. On the other hand, the ratio of stimulus-associated reward magnitudes was relatively high (5:1), which could facilitate stimulus discrimination. Furthermore, the relative simplicity of our task may contribute to the negative result observed here. Mice with ACC lesions were not impaired after a single reversal, but after serial reversals in a T-maze spatial discrimination task (Meunier et al. 1991). Thus, repeated reversals rather than a single reversal may be more appropriate to examine ACC functions. Taken together, our findings suggest that the ACC appears not to be required to discriminate similar conditioned stimuli in a simple task as used here and to use the learned significance of cues to guide instrumental behavior.

\section{Discussion}

The ACC might not be crucial in stimulus-reward learning per se, but is required if multiple stimuli must be discriminated on the basis of their associations with reward (Cardinal et al. 2002). In support of this view, lesions of the ACC impaired discrimination of reward-predictive stimuli in autoshaping (Bussey et al. 1997a; Parkinson et al. 2000; Cardinal et al. 2002, 2003) and eight-pair concurrent discrimination (Bussey et al. 1997b), but not in Pavlovian instrumental transfer (Cardinal et al. 2003). Our present findings are in keeping with this notion, as rats with ACC lesions discriminated reward-predictive stimuli during acquisition and during reversal of the original stimulus-reward magnitude contingencies. Intact performance of ACC-lesioned animals in both conditions may reflect the relative simplicity of the task involving discrimination of two stimuli only, which probably does not require a contribution of the ACC. Other prefrontal regions such as the orbitofrontal cortex (OFC) may be involved in this condition, as lesions of or an NMDA receptor blockade within the OFC affected learning of a single reversal of stimulus-reward magnitude contingencies in the task used here (Bohn et al. 2003a,b).

Requirements of tasks that challenge ACC functions are only partially known. Task complexity alone seems to not be critical. Rats with ACC lesions exhibited no performance deficits
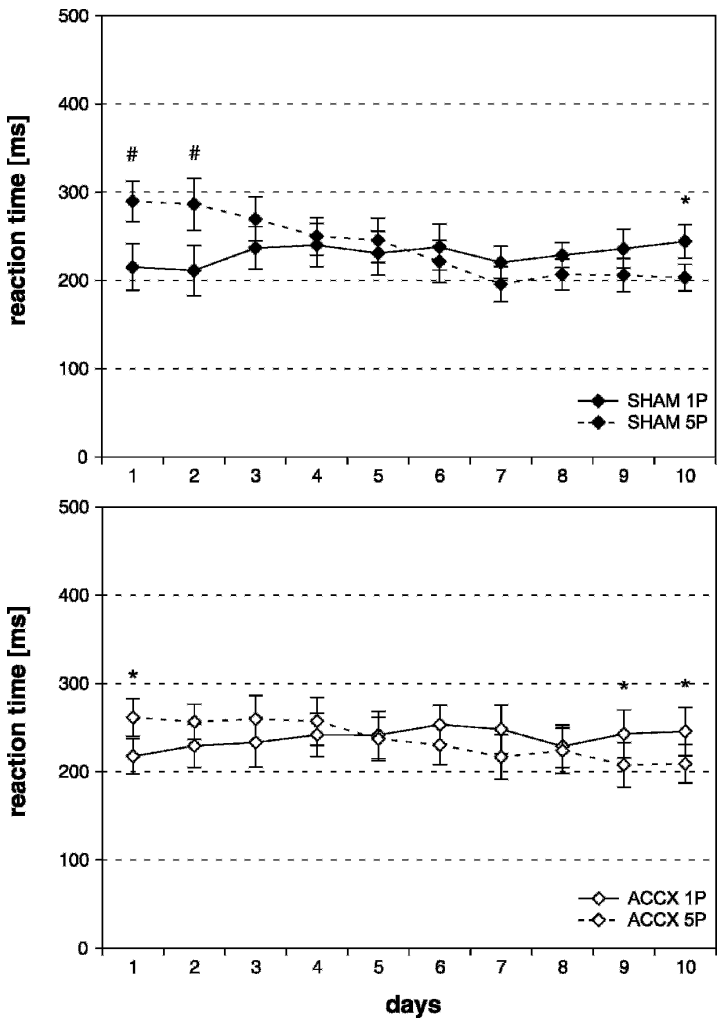

Figure 7. Effects of ACC lesions on discrimination of reward magnitude-predictive stimuli during reversal of the original stimulus-reward magnitude contingencies. Mean $( \pm$ SEM) of RTs from correct trials for expected high (five pellets) and low reward (one pellet) in sham-lesioned animals $(n=14)(A)$ and ACC-lesioned animals $(n=14)(B)$ are given. Solid line, RTs for five pellets; dotted line, RTs for one pellet. ${ }^{*} P<0.05$, $\# P<0.01$ : significant differences in RTs for expected high vs. low reward on individual days.

\section{Learning \& Memory}

www.learnmem.org 
in difficult tasks demanding a choice among delayed high reward and immediate small reward (Cardinal et al. 2001), and they even improved their performance in a few tasks, for instance a conditional visual discrimination task (Bussey et al. 1996). From our data one can infer that ACC lesions did not impair discrimination of even related stimuli which differ only in brightness, at least in a two-stimulus discrimination condition as used here. Though this implies intact stimulus perception, recent data revealed that cholinergic denervation of the ACC can impair at least some aspects of processing of sensory information and taskrelated information in a visual conditional discrimination task (Winters et al. 2004).

The ACC might also be a crucial component of a neural network encoding whether an action is worth performing given the value of the expected outcome relative to the cost of performing the action (Rushworth et al. 2004), as rats with lesions of the ACC no longer chose effortful but high-reward action in a T-maze cost-benefit task (Walton et al. 2002, 2003). Our data confirm these findings but further indicate that the ACC may not be essential for all categories of decisions that rely on cost-benefit analysis: Lesions of the ACC did not interfere with the effort a rat is willing to expend to obtain a specific reward, as indicated by the intact performance on a PR schedule. Also, choice behavior was not affected in a cost-benefit task with the options to get preferred food pellets under a PR schedule or freely available but less preferred lab chow. Notably, lesions of the ACC did not alter the preference for delayed large reward over immediate small reward in a delayed reinforcement choice task (Cardinal et al. 2001). Therefore, it has been suggested that the ACC might not be necessary when evaluating delay-based costs (Walton et al. 2003). Our observation that rats with ACC lesions still preferred food pellets under a PR schedule over freely and immediately available lab chow could be due to the fact that this condition also requires an evaluation of delay-based costs that consequently does not depend on the ACC. Under a PR schedule as used in our study, not only response costs became increasingly higher, but also the delay until reward delivery. However, this interpretation is limited, as not only timing of reward delivery and reward size differed in our task, but also the preference for the rewards, i.e., subjects had the choice between delayed access to a preferred food pellet under a PR schedule and immediate free access to less-preferred lab chow available in a large amount.

The ACC projects to the ACB and VTA (Uylings and van Eden 1990; Berendse et al. 1992; Brog et al. 1993) and receives dopaminergic afferents from the VTA (Berger et al. 1991). Therefore, the ACC could interact with these subcortical regions to mediate effort-based decisions, in particular because dopamine depletions in the ACB produced a similar shift from the HR to LR response option in the T-maze cost-benefit task (Salamone et al. 1994; Cousins et al. 1996). The finding that effort-based decisionmaking in the lever-press task was not impaired by ACC lesions as shown here but was impaired by ACB dopamine depletions (Salamone et al. 1991) does not argue against this view, because a fixed ratio (FR) schedule instead of a PR schedule was used in the latter study. As already discussed, the increasing delay until reward delivery imposed by a PR schedule may account for the lack of effect of an ACC lesion seen here. Other prefrontal regions such as the OFC may interact with the ACB in controlling such delay-based costs relative to efforts (Cardinal et al. 2004).

In conclusion, the present study demonstrates that the ACC seems not to be required to discriminate reward magnitudepredictive stimuli in a simple discrimination task, even if the stimuli are relatively similar and difficult to discriminate. Furthermore, comparative analysis of lesion effects in two different cost-benefit choice tasks revealed that the ACC is probably not necessary in all choice situations requiring an assessment of costs and benefits.

\section{Materials and Methods}

Experiments were performed according to the German Law on Animal Protection and approved by the proper authorities in Stuttgart, Germany. Three experiments were conducted; housing conditions, surgery, and histological procedures used in all experiments are given below.

\section{Animals}

Male Sprague-Dawley rats (Charles River) were used weighing $300-400 \mathrm{~g}$ at the time of surgery. They were housed in groups of up to four animals and maintained on a 12-h light/12-h dark cycle (lights on at 7:00 a.m.). Water was available ad libitum; food was restricted to $15 \mathrm{~g}$ per animal each day (Altromin).

\section{Stereotaxic surgery}

Following pretreatment with atropine sulfate $(0.5 \mathrm{mg} / \mathrm{kg}$ i.p.; Sigma-Aldrich), animals were anesthetized with sodium pentobarbital (50 mg/kg i.p., Sigma-Aldrich) and placed in a stereotaxic frame with blunt ear bars (Kopf Instruments). Standard stereotaxic methods were used for bilateral lesions at the following coordinates with reference to the atlas of Paxinos and Watson (1997): $\mathrm{AP}+1.2 \mathrm{~mm}, \mathrm{ML} \pm 0.5 \mathrm{~mm}$ and $\mathrm{DV}-3.0$ and $-2.2 \mathrm{~mm}$; $\mathrm{AP}+0.5 \mathrm{~mm}, \mathrm{ML} \pm 0.5 \mathrm{~mm}$ and $\mathrm{DV}-2.8$ and $-2.0 \mathrm{~mm}$; $\mathrm{AP}-0.2$ $\mathrm{mm}, \mathrm{ML} \pm 0.5 \mathrm{~mm}$ and $\mathrm{DV}-2.5$ and $-2.0 \mathrm{~mm}$ from the skull surface at bregma with the tooth bar $-3.3 \mathrm{~mm}$ below the interaural line. The coordinates were adapted from previous studies (Bussey et al. 1997b; Cardinal et al. 2002, 2003), and the targeted region of the ACC was the cingulate areas 1 and 2 .

Fiber-sparing excitotoxic lesions were made by infusions of quinolinic acid (Sigma-Aldrich) dissolved in $0.1 \mathrm{M}$ phosphate buffer $\left(0.07 \mathrm{M} \mathrm{Na}_{2} \mathrm{HPO}_{4}, 0.028 \mathrm{M} \mathrm{NaH}_{2} \mathrm{PO}_{4}\right.$ in bi-distilled water, sterilized by filtration) adjusted with $\mathrm{NaOH}$ to a final $\mathrm{pH}$ of 7.27.4. Sham lesions were made by infusing the phosphate buffer only. Infusions were made with a $1-\mu \mathrm{L}$ Hamilton syringe through a 25-gauge injection cannula. Infusion volume per injection was $0.5 \mu \mathrm{L}$ at each site. Infusion time was $1 \mathrm{~min}$; the injector was left in place to allow diffusion for $1 \mathrm{~min}$ at the lower injections sites and $2 \mathrm{~min}$ at the upper injections sites. After surgery, rats received a subcutaneous injection of $2.5 \mathrm{~mL}$ saline. Animals were allowed to recover for at least $7 \mathrm{~d}$.

\section{Histology}

After completion of behavioral tests, animals were killed by an overdose of Ethrane (Abbott) and perfused transcardially with $0.01 \%$ heparin sodium salt in phosphate buffered saline (PBS) followed by $4 \%$ paraformaldehyde in PBS. The brains were removed and post-fixed in paraformaldehyde overnight and dehydrated in $30 \%$ sucrose for cryoprotection. The brains were sectioned coronally $(60 \mu \mathrm{m})$ with a cryostat (Reichert Jung). Every third section was mounted on coated slides and stained with cresyl violet.

\section{Experiment 1}

\section{Subjects}

Eighteen rats were used for this experiment; one animal was discarded due to a small lesion.

\section{Apparatus}

A T-maze task involving effort-based decision-making (Salamone et al. 1994; Walton et al. 2002, 2003) was employed. The maze consisted of a start arm and two goal arms $(17 \mathrm{~cm}$ wide and $60 \mathrm{~cm}$ long); the walls were $25 \mathrm{~cm}$ high and a food well was placed at the end of each goal arm. On "forced" trials, a solid block prevented the rat from entering one goal arm. The maze and the block were made of plastic; the maze color was a uniform gray, and the block color was bright red. The barriers that the animals had to surmount were made of wire mesh in the shape of a three-dimensional right-angled triangle. The rats had to climb 
the vertical side of the triangle and descend down the slope to attain the reward. The height of the barrier was increased during training from $15 \mathrm{~cm}$ at the beginning to a final height of $25 \mathrm{~cm}$ $\left(\alpha \sim 50^{\circ}\right)$.

\section{Training}

On the first day of training, rats were placed in the start arm of the T-maze in groups of three and were allowed to explore the maze for $10 \mathrm{~min}$. The food wells were completely filled with pellets (45-mg precision pellets, Bioserv). On the second day, the rats were allowed to explore the maze individually under the same conditions as on the previous day, until all rats ingested pellets in each goal arm. Thereafter, the rats had to learn to discriminate a high-rewarded (HR, four pellets) and a low-rewarded goal arm (LR, two pellets). The position of the HR was counterbalanced for the animals, meaning that for half the animals the HR was on the right side, for the other half on the left. For the first $2 \mathrm{~d}$, the animals were allowed to sample both arms on each trial. There was an intertrial interval of at least $5 \mathrm{~min}$, and five trials per animal and day. For the next $2 \mathrm{~d}$, the access of one goal arm was prevented by placing the solid block at its entrance on each trial in a pseudorandom order, so that the rats were forced to sample one particular arm on that trial. There were 10 trials per day, five in each direction. For the next $3 \mathrm{~d}$, the rats were allowed to choose the goal arm, but were removed immediately after sampling one of the food wells (ingesting the pellets). Twelve trials per day were given; on trials 6 and 12 the block was placed in the previously chosen arm to prevent turn biases interfering with discrimination training.

After rats were choosing the HR in $>80 \%$ of the trials, a barrier of $15 \mathrm{~cm}$ height was placed in the HR arm. For this and every subsequent session, each rat ran at first one forced trial in each direction and 10 trials of free choice. The height of the barrier was increased in two steps of $5 \mathrm{~cm}$ every $3 \mathrm{~d}$, to a final height of $25 \mathrm{~cm}$.

\section{Experimental procedure}

A testing block comprised three sessions conducted on three consecutive days. The entire experiment consisted of one pre-lesion testing block and three post-lesion testing blocks. The pre-lesion block and the first and last post-lesion blocks were executed in the same manner, with one $25-\mathrm{cm}$ barrier in the HR arm. During the second post-lesion block, an identical second barrier was placed in the LR arm. The intertrial interval was at least $4 \mathrm{~min}$.

\section{Data analysis and statistics}

Choices of the HR arm on each testing day were counted and are presented as means \pm standard error of the mean (SEM). The data were subjected to a repeated-measures ANOVA with two withinsubject factors (days of testing, three and testing blocks, four) and one between-subject factor (treatment, sham vs. lesion). For post hoc comparisons of days or blocks, we used simple contrasts. All statistical computations were carried out with STATISTICA (version 5.5, StatSoft). The level of statistical significance $(\alpha$-level) was set at $P<0.05$.

\section{Experiment 2}

Thereafter animals $(n=17)$ used in Experiment 1 were subjected to Experiment 2 . They were tested first on a progressive ratio (PR) schedule and thereafter in a test in which they had the choice between pressing a lever on a PR schedule to obtain preferred food pellets or free feeding on less-preferred lab chow.

\section{Progressive ratio (PR)}

Behavioral testing was conducted in six operant test chambers (Med Associates). Each chamber was equipped with a retractable lever, a food dispenser with receptacle, an overhead house light, and two stimulus lights, one above the lever and the other above the food receptacle. An infrared photocell beam detected nosepokes into the food receptacle. The chambers were fitted in sound attenuated boxes with fans providing air circulation and a constant low-level background noise. The apparatus was con- trolled by a computer system (SmartControl-Interface and MedPC-software, Med Associates). The light above the food receptacle indicated the delivery of a food pellet in the receptacle. Subjects were habituated to a conditioned reinforcement (CRF) schedule in the operant chambers. Rats were trained for three sessions on the CRF schedule for $30 \mathrm{~min}$. Next was six sessions of an increasing fixed ratio (FR) schedule in increments of five, with three repetitions of each step (i.e. $1-1-1 ; 5-5-5 ; 10-10-10 ; .$. ) as described previously (Baunez et al. 2002). When the required FR value of each trial was achieved, the light above the food receptacle indicated the delivery of a single food pellet $(45 \mathrm{mg}$, Bioserv). The light remained on until the rat poked its nose into the receptacle. Lever presses while the light was on were counted as perseverative lever presses; they were counted but had no programmed consequences. A session lasted for $90 \mathrm{~min}$ or was ended when a rat failed to press the lever for consecutive $5 \mathrm{~min}$. For each session, the value of the last completed ratio (breaking point) was recorded, as well as the amount of received rewards, the perseverative lever presses, and the duration of the session.

\section{Cost-benefit choice test}

In this task the rats had the choice between working for their preferred food (Bioserv pellets) by pressing the lever on a PR schedule as described above or obtaining lab chow (about $15 \mathrm{~g}$ ) being freely available in a dish within the operant chamber. The food receptacle and the lever were positioned on the same wall of the operant chamber, and the dish containing the lab chow was situated in a corner on the opposite side of the operant chamber. A session lasted for $90 \mathrm{~min}$ or ended when a rat failed to press the lever for consecutive $5 \mathrm{~min}$. Thereafter the amount of lab chow ingested was calculated.

\section{Data analysis and statistics}

PR test data were analyzed using a repeated-measures ANOVA with breaking point (perseverative lever presses or duration of session) and days as within-subject factors and treatment as between-subject factor (sham vs. lesion). When significant effects were detected, post hoc comparisons were made using simple contrast analysis. Data from the cost-benefit choice task were analyzed separately. The breaking points from the choice task and breaking points from the last day of the progressive ratio task were compared. Lever presses were subjected to an ANOVA with treatment and test condition as between-subject factors. Ingested amounts of lab chow were analyzed by a $t$-test for independent samples.

\section{Experiment 3}

Twenty-eight rats were used in Experiment 3.

\section{Reaction time task}

Behavioral testing was conducted in the operant test chambers described above. This experiment uses a reaction time task (Giertler et al. 2003) demanding conditioned lever release with instructive stimuli indicating the reward magnitude to be obtained after a subsequent imperative stimulus. A rat had to press the lever and wait for the imperative stimulus that was provided by the stimulus light above the lever after a period of $0.3 \mathrm{sec}$. The imperative stimulus signaled the rat to release the lever quickly and to respond to the food receptacle in which the food pellets were delivered (45-mg pellets, Bioserv). On each correct trial, a rat received either one or five food pellets. The number of pellets for each trial was pseudo-randomly determined in advance and signaled to the rat by two distinct brightness levels of the cue lights, which provided the instructive stimuli. After the intertrial interval of $3 \mathrm{sec}$, the instructive stimulus was turned on at the beginning of each trial $3 \mathrm{sec}$ before lever insertion and remained present until delivery of the food reward. To check for equal perception of instructive stimuli of the two different brightness levels, for $50 \%$ of the rats, a bright stimulus was associated with delivery of five pellets, and a dim stimulus was associated with delivery of one pellet. For the other $50 \%$ of the rats, the opposite pattern was used. 
Reaction time (RT) was defined as latency from the onset of the imperative stimulus to lever release and was recorded with an accuracy of $<10 \mathrm{msec}$. For a correct trial, animals had to release the lever within RT $<2$ sec after presentation of the imperative stimulus. Responses before onset of the imperative stimulus presentation were defined as "early" responses, and responses with RT $\geq 2$ sec were defined as "late" responses. A daily individual session demanded 60 correct trials, i.e., 30 correct trials for each reward magnitude (one and five pellets).

\section{Experimental procedure}

\section{Preoperative habituation}

Initially, rats were habituated to the operant chamber with access to food pellets in the food receptacle, followed by a habituation program on a CRF schedule conducted until all rats attained the criterion of 20 consecutive lever presses within a session. Afterwards, rats were subjected to surgery.

\section{Acquisition}

Rats were trained for 14 sessions on the RT task. During acquisition, responses were guided by stimulus-associated reward magnitudes, i.e., mean RTs of responses associated with low reward were significantly longer than those associated with high reward.

\section{Reversal}

Learning a reversal of original stimulus-reward magnitude contingencies was tested for another 10 sessions, i.e., rats had to learn that the stimulus formerly associated with high reward was now predicting low reward, and vice versa.

\section{Data analysis and statistics}

Brightness levels of the instructive stimulus were perceived equally, as shown in earlier studies (Bohn et al. 2003b): For a given reward magnitude level, mean accuracy and RT values obtained with a bright or a dim stimulus did not differ significantly (data not shown). Therefore, response measures for a given reward magnitude obtained with bright and dim instructive stimuli were pooled. Accuracy of performance was determined by the proportion of correct trials from the overall number of trials (early + correct + late) necessary to reach the criterion of 30 correct responses for each stimulus-reward magnitude relationship ([correct responses/early + correct + late responses] $\times 100$ ).

The calculations on RT performance were conducted with data from correct trials ( $\mathrm{RT}<2 \mathrm{sec}$ ). When averaging RT data, a geometric mean was calculated for each rat for each session, as the geometric mean is less influenced by outlying data points than is the arithmetic mean. Overall, RT means of responses associated with the high- and low-reward magnitude represent the arithmetic average of the geometric means of individual rats (Brasted et al. 1997).

Treatment effects on accuracy of performance, early and late responses, and RT of correct responses were assessed by betweensubjects comparisons of groups treated with vehicle or QA. Data were analyzed using an ANOVA with treatment as betweensubjects factor, reward magnitude and days as within-subjects (repeated measures) factors, followed by planned contrast analyses. Accuracy of performance, early and late responses, and RTs of correct responses during reversal were compared using a separate ANOVA followed by planned contrast analyses.

\section{Acknowledgments}

This research was supported by the Deutsche Forschungsgemeinschaft (DFG Ha2340/5-1).

\section{References}

Baunez, C., Amalric, M., and Robbins, T.W. 2002. Enhanced food-related motivation after bilateral lesions of the subthalamic nucleus. J. Neurosci. 22: 562-568.

Berendse, H.W., Galis-de Graaf, Y., and Groenewegen, H.J. 1992. Topographical organization and relationship with ventral striatal compartments of prefrontal corticostriatal projections in the rat. $J$. Comp. Neurol. 316: 314-347.
Berger, B., Gaspar, P., and Verney, C. 1991. Dopaminergic innervation of the cerebral cortex: Unexpected differences between rodents and primates. Trends Neurosci. 14: 21-27.

Bohn, I., Giertler, C., and Hauber, W. 2003a. Orbital prefrontal cortex and guidance of instrumental behaviour in rats under reversal conditions. Behav. Brain Res. 143: 49-56.

Bohn, I., Giertler, C., and Hauber, W. 2003b. NMDA receptors in the rat orbital prefrontal cortex are involved in guidance of instrumental behaviour under reversal conditions. Cereb. Cortex 13: 968-976.

Bowman, E.M. and Brown, V.J. 1998. Effects of excitotoxic lesions of the rat ventral striatum on the perception of reward cost. Exp. Brain Res. 123: 439-448.

Brasted, P.J., Humby, T., Dunnett, S.B., and Robbins, T.W. 1997. Unilateral lesions of the dorsal striatum in rats disrupt responding in egocentric space. J. Neurosci. 17: 8919-8926.

Brog, J.S., Salyapongse, A., Deutch, A.Y., and Zahm, D.S. 1993. The patterns of afferent innervation of the core and shell in the accumbens part of the rat ventral striatum-Immunohistochemical detection of retrogradely transported fluoro-gold. J. Comp. Neurol. 338: 255-278.

Bussey, T.J., Muir, J.L., Everitt, B.J., and Robbins, T.W. 1996. Dissociable effects of anterior and posterior cingulate cortex lesions on the acquisition of a conditional visual discrimination: Facilitation of early learning vs. impairment of late learning. Behav. Brain Res. 82: $45-56$.

Bussey, T.J., Everitt, B.J., and Robbins, T.W. 1997a. Dissociable effects of cingulate and medial frontal cortex lesions on stimulus-reward learning using a novel Pavlovian autoshaping procedure for the rat: Implications for the neurobiology of emotion. Behav. Neurosci. 111: 908-919.

Bussey, T.J., Muir, J.L., Everitt, B.J., and Robbins, T.W. 1997b. Triple dissociation of anterior cingulate, posterior cingulate, and medial frontal cortices on visual discrimination tasks using a touchscreen testing procedure for the rat. Behav. Neurosci. 111: 920-936.

Cardinal, R.N., Pennicott, D.R., Sugathapala, C.L., Robbins, T.W., and Everitt, B.J. 2001. Impulsive choice induced in rats by lesions of the nucleus accumbens core. Science 292: 2499-2501.

Cardinal, R.N., Parkinson, J.A., Lachenal, G., Halkerston, K.M., Rudarakanchana, N., Hall, J., Morrison, C.H., Howes, S.R., Robbins, T.W., and Everitt, B.J. 2002. Effects of selective excitotoxic lesions of the nucleus accumbens core, anterior cingulate cortex, and central nucleus of the amygdala on autoshaping performance in rats. Behav. Neurosci. 116: 553-567.

Cardinal, R.N., Parkinson, J.A., Marbini, H.D., Toner, A.J., Bussey, T.J., Robbins, T.W., and Everitt, B.J. 2003. Role of the anterior cingulate cortex in the control over behavior by pavlovian conditioned stimuli in rats. Behav. Neurosci. 117: 566-587.

Cardinal, R.N., Winstanley, C.A., Robbins, T.W., and Everitt, B.J. 2004. Limbic corticostriatal systems and delayed reinforcement. Ann. NY Acad. Sci. 1021: 33-50.

Cousins, M.S., Atherton, A., Turner, L., and Salamone, J.D. 1996. Nucleus accumbens dopamine depletions alter relative response allocation in a T-maze cost/benefit task. Behav. Brain Res. 74: 189-197.

Divac, I. and Diemer, N.H. 1980. Prefrontal system in the rat visualized by means of labeled deoxyglucose-Further evidence for functional heterogeneity of the neostriatum. J. Comp. Neurol. 190: 1-13.

Giertler, C., Bohn, I., and Hauber, W. 2003. The rat nucleus accumbens is involved in guiding of instrumental responses by stimuli predicting reward magnitude. Eur. J. Neurosci. 18: 1993-1996.

Hadland, K.A., Rushworth, M.F., Gaffan, D., and Passingham, R.E. 2003. The anterior cingulate and reward-guided selection of actions. $J$. Neurophysiol. 89: 1161-1164.

Hodos, W. 1961. Progressive ratio as a measure of reward strength. Science 134: 943-944.

McGeorge, A.J. and Faull, R.L. 1989. The organization of the projection from the cerebral cortex to the striatum in the rat. Neuroscience 29: $503-537$.

Meunier, M., Jaffard, R., and Destrade, C. 1991. Differential involvement of anterior and posterior cingulate cortices in spatial discriminative learning in a T-maze in mice. Behav. Brain Res. 44: 133-143.

Parkinson, J.A., Willoughby, P.J., Robbins, T.W., and Everitt, B.J. 2000. Disconnection of the anterior cingulate cortex and nucleus accumbens core impairs Pavlovian approach behavior: Further evidence for limbic cortical-ventral striatopallidal systems. Behav. Neurosci. 114: 42-63.

Paxinos, G. and Watson, C. 1997. The rat brain in stereotaxic coordinates, 3rd ed. Academic Press, Inc., San Diego, CA.

Rushworth, M.F., Walton, M.E., Kennerley, S.W., and Bannerman, D.M. 2004. Action sets and decisions in the medial frontal cortex. Trends Cogn. Sci. 8: 410-417.

Salamone, J.D., Steinpreis, R.E., McCullough, L.D., Smith, P., Grebel, D., 
and Mahan, K. 1991. Haloperidol and nucleus accumbens dopamine depletion suppress lever pressing for food but increase free food consumption in a novel food choice procedure. Psychopharmacology (Berl.) 104: 515-521.

Salamone, J.D., Cousins, M.S., and Bucher, S. 1994. Anhedonia or anergia? Effects of haloperidol and nucleus accumbens dopamine depletion on instrumental response selection in a T-maze cost/benefit procedure. Behav. Brain Res. 65: 221-229.

Salamone, J.D., Cousins, M.S., and Snyder, B.J. 1997. Behavioral functions of nucleus accumbens dopamine: Empirical and conceptual problems with the anhedonia hypothesis. Neurosci. Biobehav. Rev. 21: 341-359.

Salamone, J.D., Correa, M., Mingote, S., and Weber, S.M. 2003. Nucleus accumbens dopamine and the regulation of effort in food-seeking behavior: Implications for studies of natural motivation, psychiatry, and drug abuse. J. Pharmacol. Exp. Ther. 305: 1-8.

Shima, K. and Tanji, J. 1998. Role for cingulate motor area cells in voluntary movement selection based on reward. Science 282: $1335-1338$.
Uylings, H.B. and van Eden, C.G. 1990. Qualitative and quantitative comparison of the prefrontal cortex in rat and in primates, including humans. Prog. Brain Res. 85: 31-62.

Walton, M.E., Bannerman, D.M., and Rushworth, M.F. 2002. The role of rat medial frontal cortex in effort-based decision making. J. Neurosci. 22: 10996-11003.

Walton, M.E., Bannerman, D.M., Alterescu, K., and Rushworth, M.F. 2003. Functional specialization within medial frontal cortex of the anterior cingulate for evaluating effort-related decisions. J. Neurosci. 23: $6475-6479$.

Winters, B.D., Robbins, T.W., and Everitt, B.J. 2004. Selective cholinergic denervation of the cingulate cortex impairs the acquisition and performance of a conditional visual discrimination in rats. Eur. J. Neurosci. 19: 490-496.

Received December 8, 2004; accepted in revised form March 29, 2005.

\section{Learning \& Memory}




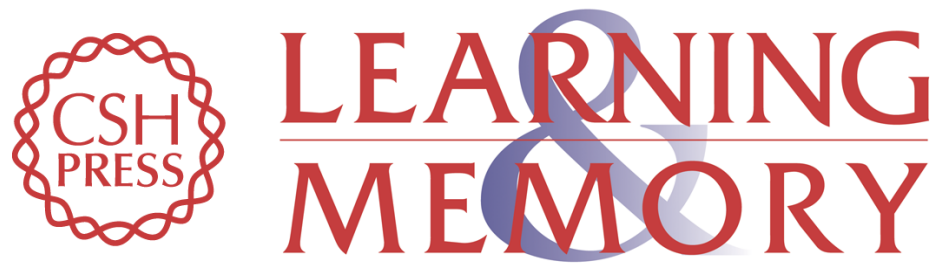

\section{Involvement of the rat anterior cingulate cortex in control of instrumental responses guided by reward expectancy}

Judith Schweimer and Wolfgang Hauber

Learn. Mem. 2005, 12:

Access the most recent version at doi:10.1101//m.90605

References This article cites 33 articles, 8 of which can be accessed free at:

http://learnmem.cshlp.org/content/12/3/334.full.html\#ref-list-1

License

Email Alerting Receive free email alerts when new articles cite this article - sign up in the box at the Service top right corner of the article or click here. 